\title{
Effect of Variation in Voltage on the Synthesis of Ag Nanopowder by Pulsed Wire Evaporation
}

\author{
Joon-Woo Song, ${ }^{1}$ Dong-Jin Lee, ${ }^{2}$ Fikret Yilmaz, ${ }^{3}$ and Soon-Jik Hong ${ }^{1}$ \\ ${ }^{1}$ Division of Advanced Materials Engineering and Institute for Rare Metals, Kongju National University, 275 Budae-Dong, Chungnam \\ Cheonan 330-717, Republic of Korea \\ ${ }^{2}$ Nano Technology Inc., 290-19 Daehwa, Daedeok, Daejeon Metropolitan City 306-801, Republic of Korea \\ ${ }^{3}$ Department of Physics, Faculty of Art and Science, Gaziosmanpasa University, Tasliciftlik Campus, 60240 Tokat, Turkey
}

Correspondence should be addressed to Soon-Jik Hong, hongsj@kongju.ac.kr

Received 7 November 2011; Accepted 22 December 2011

Academic Editor: Kin Tak Lau

Copyright ( $) 2012$ Joon-Woo Song et al. This is an open access article distributed under the Creative Commons Attribution License, which permits unrestricted use, distribution, and reproduction in any medium, provided the original work is properly cited.

\begin{abstract}
Nanosized Ag particles were obtained in liquid medium, which is composed of deionized water and sodium hexametaphosphate, using pulsed wire evaporation within hundreds of microseconds from pure initial Ag wire (99.98\%), with varying voltage. Changes in powder morphology and microstructure due to change in supply voltage was observed by FE-SEM and HR-TEM, and phase structure of powders was analyzed by XRD. The results revealed that average particle size slightly increased with increasing supply voltage without any impurity and oxide phase, which was explained in terms of addition of stabilizer into the coolant liquid. The experimental results also suggest that addition of sodium hexametaphosphate into the coolant liquid is good for enhancing the dispersibility property of silver nanopowders.
\end{abstract}

\section{Introduction}

Nanosize particles (nanopowders) have attracted a great deal of attention owing to their significant applications in nanotechnology and nanobiotechnology. Therefore, scientists have shown great interest to discover new production methods and new application fields of nanopowders [1]. Different methods for fabrication of metal nanopowders have been introduced in recent years, which are often classified into three groups: mechanical process; physical process; chemical process [2]. The chemical process has been widely adopted in production of metal nanopowders. While using chemical process, however, undesired products could be formed during chemical reaction [3]. Quite recently, pulsed wire evaporation (PWE) technique, which is a physical process that allows high production rate and particle size control with high efficiency, has been introduced to produce metal nanopowders. In this technique, a high power pulsed dc current passing through a thin metal wire leads to the wire explosion, and the large amount of heat energy causes the wire to melt, followed by subsequent evaporation and formation of plasma. The plasma formed during the process expands and cools when it interacts with a coolant such as an inert gas or liquid, and then nanoparticles are formed through the nucleation process $[2,4]$. Nowadays, the PWE technique can be used effectively to fabricate metal nanopowders, namely, $\mathrm{Ni}$ [2], $\mathrm{Fe}$ [3], $\mathrm{Al}$ [4], $\mathrm{Cu}$ [5], Ag [6], and so forth, or nano-powder alloys, such as Pt-Ni [7], Cu$\mathrm{Ni}$ [8], and Cu-Ni-P [9]. Among the metal nanopowders, Ag nanopowders have drawn considerable interest and been used in widespread fields, including catalysis, photography, nanophotocatalysis in cosmetics, food, medicine, and antibacterial agents in clothes $[1,6]$.

Several parameters in PWE such as energy deposition, polarity of charging voltage, type of coolant, wire diameter, and feeding distance can influence the size and morphology of the particles. In this study, we therefore aimed to find out the effects of voltage level on particle size and morphology of silver nanopowders produced by the PWE process in which pure water + sodium hexametaphosphate $\left(\mathrm{NaPO}_{3}\right)_{6}$ was used as coolant liquid. 


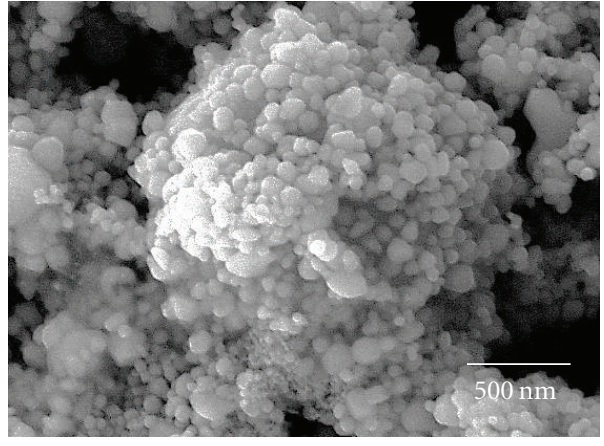

(a)

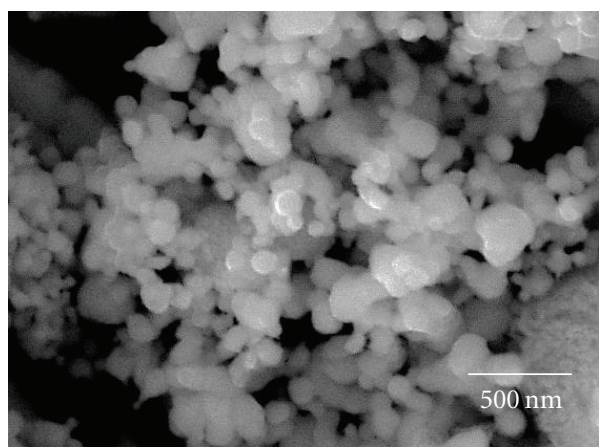

(c)

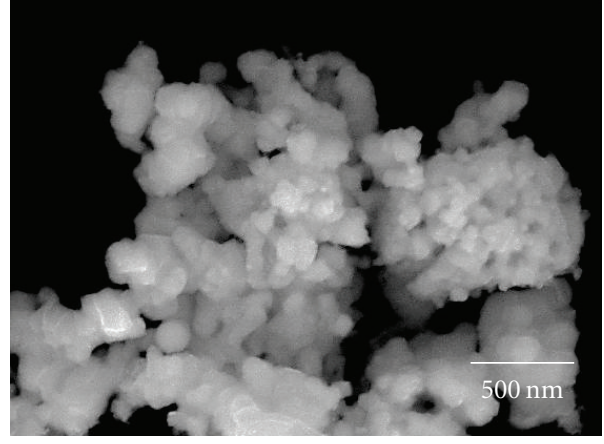

(b)

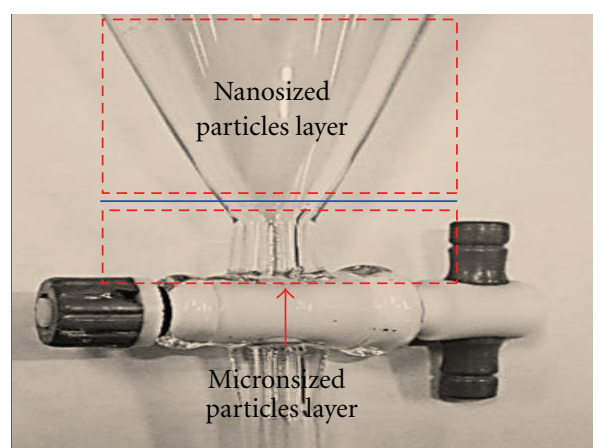

(d)

FIGURE 1: FE-SEM micrographs of PWE-ed Ag powder for various supply voltages (a) $210 \mathrm{~V}$, (b) $260 \mathrm{~V}$, (c) $310 \mathrm{~V}$, and (d) the funnel used to collect silver particles shows nano- and micron-sized layers after drying process.

\section{Experimental Procedure}

In this paper, silver nanopowders with purity of $99.99 \%$ were produced by pulsed wire evaporation (PWE) in liquid medium. The maximum power and energy consumption of PWE apparatus are $100 \mathrm{~W}$ and $110 \mathrm{~J}$, respectively. The diameter of silver wire was $0.2 \mathrm{~mm}$, and feeding length was $80 \mathrm{~mm}$. The experiments were carried out with the capacitance of $2200 \mu \mathrm{F}$ for supply voltages of 210,260 , and 310 V. 500 explosions were done for each experiment and manufactured powders were collected in coolant liquid. The coolant liquid was prepared from deionized (DI) water with the addition of $1 \mathrm{wt}$. \% $\left(\mathrm{NaPO}_{3}\right)_{6}$ (sodium hexametaphosphate). As-received suspensions were dried in a funnel at $30^{\circ} \mathrm{C}$. Crystal structure, particles size, and morphology of powders were studied by X-ray diffraction (XRD), fieldemission scanning electron microscopy (FE-SEM), and highresolution transmission electron microscopy (HR-TEM).

\section{Results and Discussion}

Our primary purpose in this paper is to examine Nanosized particles rather than micronsized particles because the product fabricated are largely composed of Nanosized particles being $95 \%$ of total products. We think that, however, to examine the shape and size of the submicron/micron-sized particles as well as Nanosized particles may be beneficial for practical applications. We used the funnel shown in Figure $1(\mathrm{~d})$ to dry the Ag powders. Due to the fact that submicron and/or micron-sized particle are heavier than Nanosized particle, submicron and/or micron-sized particles in Ag suspension are expected to sink through the bottom of the bottle, while Nanosized particles accumulate at the top. The particles deposited at the top and the bottom region of the funnel were used for HR-TEM and FE-SEM observations, respectively. Figures $1(\mathrm{a})-1(\mathrm{c})$ show the FESEM micrographs of bottom region layer of Ag particles produced with varied supply voltage. From the results of FE-SEM, it was found that particles are largely composed of Nanosized particles and some submicron-sized particles rather than micron-sized particles. Mean particle size was found to be around $100 \mathrm{~nm}$ for supply voltage of $210 \mathrm{~V}$, and a slight increase was observed in particles size with increasing supply voltage. The silver particles are nearly spherical in shape for all conditions. A clear agglomeration behavior between particles can be seen for all conditions, but the agglomeration tendency with increasing supply voltage is not distinct for the particles received from the bottom side of the funnel.

Figure 2 shows the XRD patterns of silver nanopowders taken from the top of the funnel. The clear peaks corresponding to Ag phase was crystalline with fcc structure detected for all conditions without any impurity or oxide phase. Sharpness and intensity of the peaks increase steadily with increasing supply voltage, which can be ascribed by an increase in particle size. The particle sizes were calculated from the full-width half-maximum (FWHM) of 
TABLE 1: Particle size of nanopowders calculated using X-ray peak broadening and TEM observations.

\begin{tabular}{|c|c|c|c|c|}
\hline Supply voltage (V) & Particle size from XRD $(\mathrm{nm})$ & Particle size from TEM (nm) & Particle morphology & Dispersibility \\
\hline 210 & 39.9 & $12.2 \pm 3.4$ & Spherical & Good \\
\hline 260 & 52.5 & $19.8 \pm 4.4$ & Spherical & Not good \\
\hline 310 & 55.9 & $22.7 \pm 5.6$ & Spherical & Not good \\
\hline
\end{tabular}

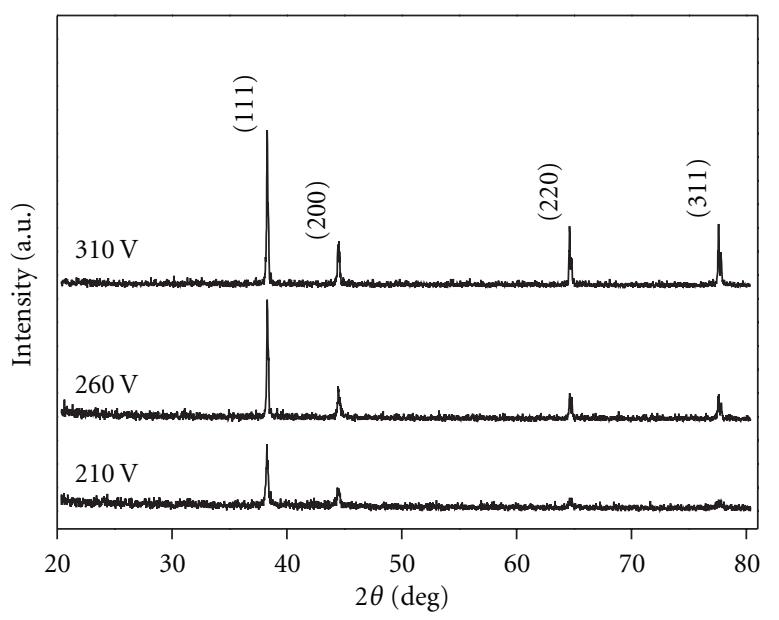

FIGURE 2: XRD patterns of Ag nanopowders fabricated by pulsed wire evaporation (PWE).

the peak (111), using the classical Scherrer equation, $d=$ $0.9 \lambda / \beta \cos \theta$, where $d$ is the particle size, $\beta$ is the FWHM value, and $\lambda$ is the wavelength of the -ray $(\lambda \approx 0.154 \mathrm{~nm})$ and estimated to be $39.9,52.5$, and $55.9 \mathrm{~nm}$ for 210,260 , and $310 \mathrm{~V}$ (Table 1), respectively.

High-resolution TEM image of silver nanopowders produced at different supply voltage is presented in Figure 3. The figure indicates that the powders, for the supply voltage of 210 volt, are well dispersed and of spherical shape (Figures $3(\mathrm{a})$ and $3(\mathrm{~b})$ ). As the supply voltage increases, although the particles still keep their spherical shape, some irregular round particles become visible as well as dislocations and agglomerates (Figures 3(c)-3(f)). The agglomeration tendency of these particles, however, slightly differs from the behavior suggested in theoretical terms [6]. In general, the dispersibility of nanoparticles has been considered as an important factor in application of nanopowders because the agglomeration between nanoparticles deteriorates the catalytic activity [2]. We think that chemical agent addition $\left(\mathrm{NaPO}_{3}\right)_{6}$, into the coolant water, may enhance the dispersibility of these nanoparticles. Figure 4 shows the TEM micrographs of Nanosized powder obtained with and without stabilizer for the voltage level of $310 \mathrm{~V}$. It is clearly seen that the powders obtained without stabilizer has strong agglomerated clusters (Figure 4(a)) while the other consists of well dispersed particles (Figure 4(b)).

The particle size of Ag nanopowders was estimated to be $13.0 \pm 1.0,16.7 \pm 3.0$, and $24.5 \pm 4.0$ by HR-TEM for the supply voltages of 210,260 , and $310 \mathrm{~V}$, respectively (Table 1 ). Same tendency in particle size is seen clearly with the one calculated from XRD pattern, in which particle size increases with increasing supply voltage. The standard deviation of average particle size increases with increasing supply voltage indicating that particle size distribution range became wider for higher voltage level. No micron-sized particle, which is originated from the liquid droplets or strongly agglomerated particles, was observed. The results denote that average particle size increases with increasing supply voltage, which is opposite to our expectation. Kwon et al. reported that super heating factor or specific energy input into wire is the main parameter influencing the powder properties such as morphology, surface modification, and particle size [8]. They also revealed that particle size decreases with increasing the super heating factor and the specific energy input into the wire. Similarly, Jung et al. observed that an increase in voltage reduced the particle size of Ni nanopowders, which seconds the above explanation [2]. We think that the differences between our results and literature may be related to the following reasons.

3.1. Temperature of Coolant Medium. Yun et al. reported that variation of the average particle size and particle size distribution depended on the medium temperature [6]. They found that average size of $\mathrm{Ag}$ particles increased with increasing temperature and the average particle size changed from $37 \mathrm{~nm}$ to $54 \mathrm{~nm}$ for temperature of $20^{\circ} \mathrm{C}$ and $80^{\circ} \mathrm{C}$, respectively. In this study, all experiments were launched in a fixed medium temperature, $24^{\circ} \mathrm{C}$. While the experiment was being in progress, some thermal energy, which was generated by the wire explosion, could be absorbed by the medium, and this could result in a temperature increase. Since the thermal energy is related to input energy, it is expected to have an increase in the medium temperature with increasing of supply voltage. However, the increasing in temperature should not be so much owing to the fact that the explosion occurred in a very short time and energy exchange between coolant and ambience is continuously going on during experiment. In our experiments, we dedicated a little temperature increase, $1-2^{\circ} \mathrm{C}$, depending on the supply voltage. As a result, increase in particle size can be related to medium temperature, but the effect of medium temperature is negligible in this study.

3.2. Energy Dissipation in Wire. Energy dissipation in the wire is another factor that could affect our results. If deionized (DI) water is used as coolant liquid, the electrical energy is dissipated mainly in the wire, as its resistivity is very low compared to DI water [10]. In this study, we added 1 wt. \% sodium hexametaphosphate into the DI water. Due to its easy solubility in water, sodium hexametaphosphate 


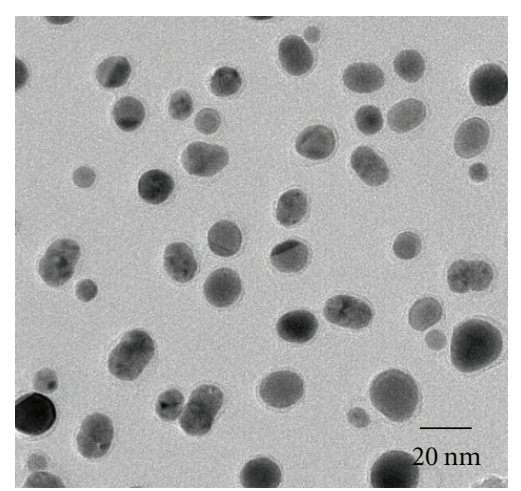

(a)

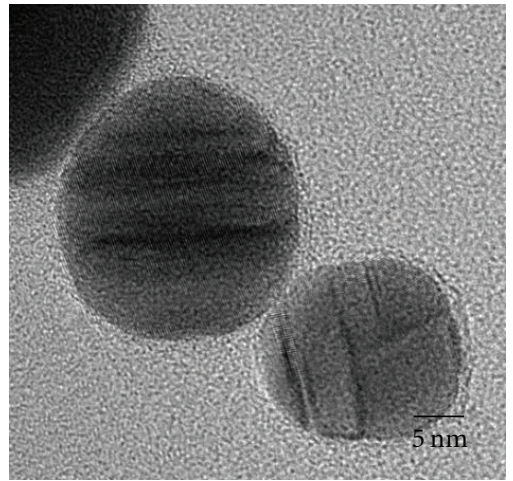

(d)

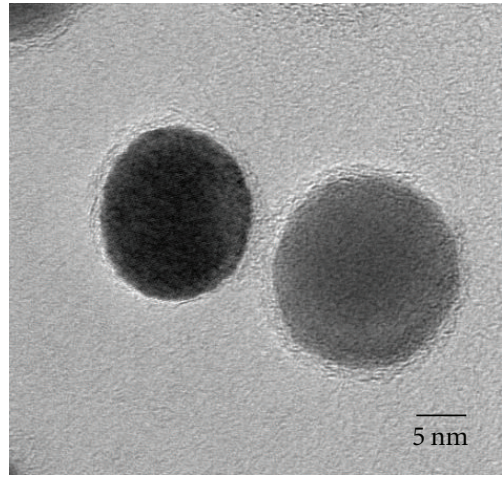

(b)

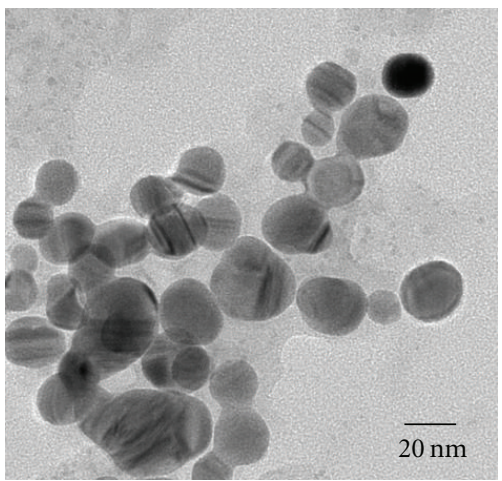

(e)

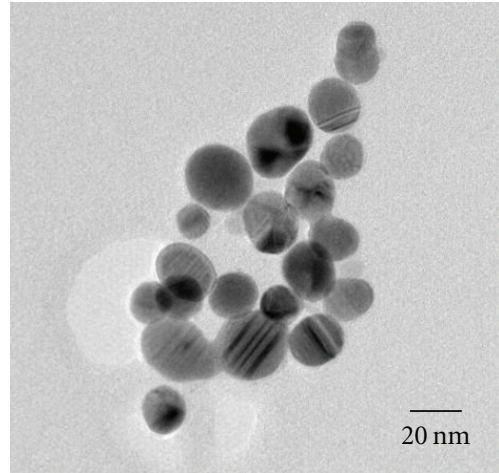

(c)

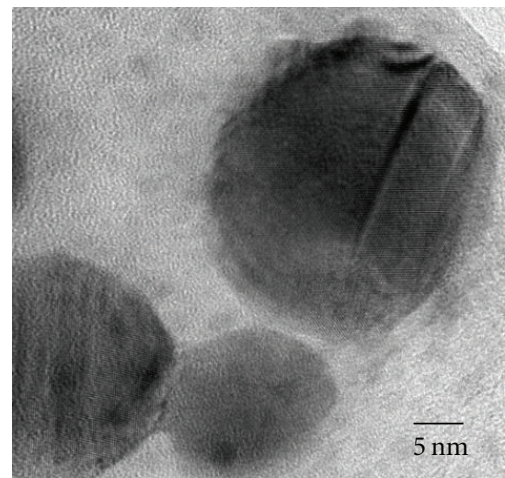

(f)

FIGURE 3: Low- and high-magnification TEM micrographs of Ag nano-powder for different supply voltage (a-b) $210 \mathrm{~V}$, (c-d) $260 \mathrm{~V}$, and (e-f) $310 \mathrm{~V}$.

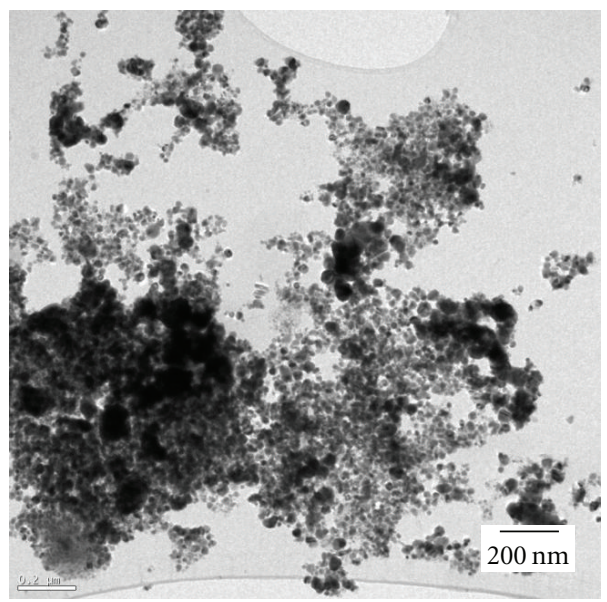

(a)

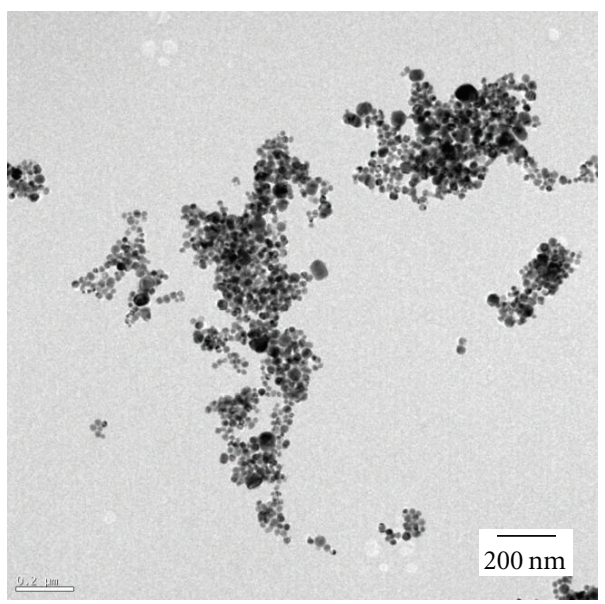

(b)

FIGURE 4: Nanosized particles obtained for the voltage level of $310 \mathrm{~V}$ show dispersibility behavior (a) without stabilizer and (b) with stabilizer.

is mainly used as a water softening agent for hard water. It ionizes to give sodium cation and complex anion as indicated in the formula below:

$$
\left(\mathrm{NaPO}_{3}\right)_{6} \longrightarrow 2 \mathrm{Na}^{+}+\mathrm{Na}_{4} \mathrm{P}_{6} \mathrm{O}_{18}^{-2} .
$$

Addition of sodium hexametaphosphate into the DI water increases the conductivity of water. In other words, it reduces its resistivity so that the electrical energy can dissipate in the wire and the water as well. Referring to Figure 5, it is obviously seen that the energy consumption of the wire no longer changes with increasing supply voltage, which means that a certain amount of energy is dissipated in the water. Cho et al. also suggested that longer plasma formation time led to a much higher energy deposition in wire and thus 


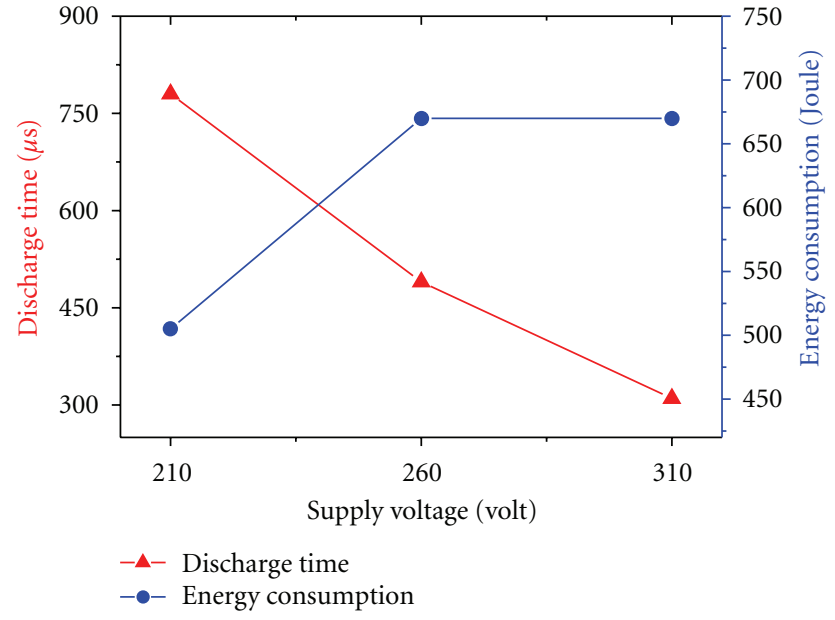

Figure 5: Changes in energy consumption and discharge time during the process for different supply voltages.

causes a decrease in particle size [10]. So the longer discharge time for supply voltage of $210 \mathrm{~V}$ could result in a much higher energy deposition in the wire, decreasing the particle size. As can be seen from Figure 5, the discharge time reduces with increasing supply voltage, and as a result the particle size increases, which confirms our approximations. Although energy consumptions for 260 and $310 \mathrm{~V}$ are approximately the same, discharge time is decreasing with increasing voltage.

It must be noted that certain chemical agents may react with Ag ions and result in silver complexes such as ammonia, amine, cyanide, halide, olefin, sulfur, and some organic complexes [11]. In our experiments, however, reaction between $\mathrm{Ag}$ ions and the ions indicated in (1) have low probability. Moreover, we determined the $\mathrm{pH}$ level of the silver suspensions to be $6.9,6.3$, and 6.5 for supply voltages of 210,260 , and 310 , respectively. These results are comparable to the $\mathrm{pH}$ level of starting coolant liquid, 6.8. The $\mathrm{pH}$ measurements also confirm that there is no chemical reaction between silver ions and coolant ions.

\section{Conclusions}

We have successfully synthesized silver nanopowders using pulsed wire explosion process in DI water with addition of sodium hexametaphosphate. Effects of supply voltage and coolant medium on the dispersibility and the particle size have been studied. Well-dispersed Ag nanoparticles without unwanted products could be obtained. Most of these Ag particles exhibited almost spherical shape and very small particle size (less than $30 \mathrm{~nm}$ ). The average particle size increased with increasing the supply voltage. The major reason is that addition of sodium hexametaphospate into DI water increases the conductivity of liquid, and thus the particles size increases due to the decrease in energy dissipation in $\mathrm{Ag}$ wire during the explosion process.

\section{References}

[1] D. C. Tien, C. Y. Liao, J. C. Huang et al., "Novel technique for preparing a nano-silver water suspension by the arc-discharge method," Reviews on Advanced Materials Science, vol. 18, no. 8, pp. 752-758, 2008.

[2] T. K. Jung, D. W. Joh, H. S. Lee, and M. H. Lee, "Fabrication of $\mathrm{Ni}$ nanopowder using wire explosion process and its characterization," Reviews on Advanced Materials Science, vol. 28, no. 2, pp. 171-174, 2011.

[3] H. M. Lee, Y. R. Uhm, and C. K. Rhee, "Phase control and characterization of Fe and Fe-oxide nanocrystals synthesized by pulsed wire evaporation method," Journal of Alloys and Compounds, vol. 461, no. 1-2, pp. 604-607, 2008.

[4] J. K. Antony, N. J. Vasa, S. R. Chakravarthy, and R. Sarathi, "Understanding the mechanism of nano-aluminum particle formation by wire explosion process using optical emission technique," Journal of Quantitative Spectroscopy and Radiative Transfer, vol. 111, no. 17-18, pp. 2509-2516, 2010.

[5] P. K. Dash and Y. Balto, "Generation of nano-copper particles through wire explosion method and its characterization," Research Journal of Nanoscience and Nanotechnology, vol. 1, no. 1, pp. 25-33, 2011.

[6] G. S. Yun, L. H. Bac, J. S. Kim, Y. S. Kwon, H. S. Choi, and J. C. Kim, "Preparation and dispersive properties of Ag colloid by electrical explosion of wire," Journal of Alloys and Compounds, vol. 509, supplement 1, pp. S348-S352, 2011.

[7] T. K. Jung, D. W. Joh, H. S. Lee, and M. H. Lee, "Fabrication of $\mathrm{Ni}, \mathrm{Pt}, \mathrm{Pt} / \mathrm{Ni}$ nano powders using wire explosion process and characterization," Procedia Engineering, vol. 10, no. 2011, pp. 728-733, 2011.

[8] Y. S. Kwon, V. V. An, A. P. Ilyin, and D. V. Tikhonov, "Properties of powders produced by electrical explosions of copper-nickel alloy wires," Materials Letters, vol. 61, no. 14-15, pp. 3247-3250, 2007.

[9] W. Kim, J. S. Park, C. Y. Suh, J. G. Ahn, and J. C. Lee, "Cu-Ni$\mathrm{P}$ alloy nanoparticles prepared by electrical wire explosion," Journal of Alloys and Compounds, vol. 465, no. 1-2, pp. L4-L6, 2008.

[10] C. Cho, Y. W. Choi, C. Kang, and G. W. Lee, "Effects of the medium on synthesis of nanopowders by wire explosion process," Applied Physics Letters, vol. 91, no. 14, Article ID 141501, 3 pages, 2007.

[11] R. E. Kirk and D. F. Othmer, Kirk-Othmer Encyclopedia of Chemical Technology, John Wiley and Sons, Hoboken, NJ, USA, 5th edition, 2007. 

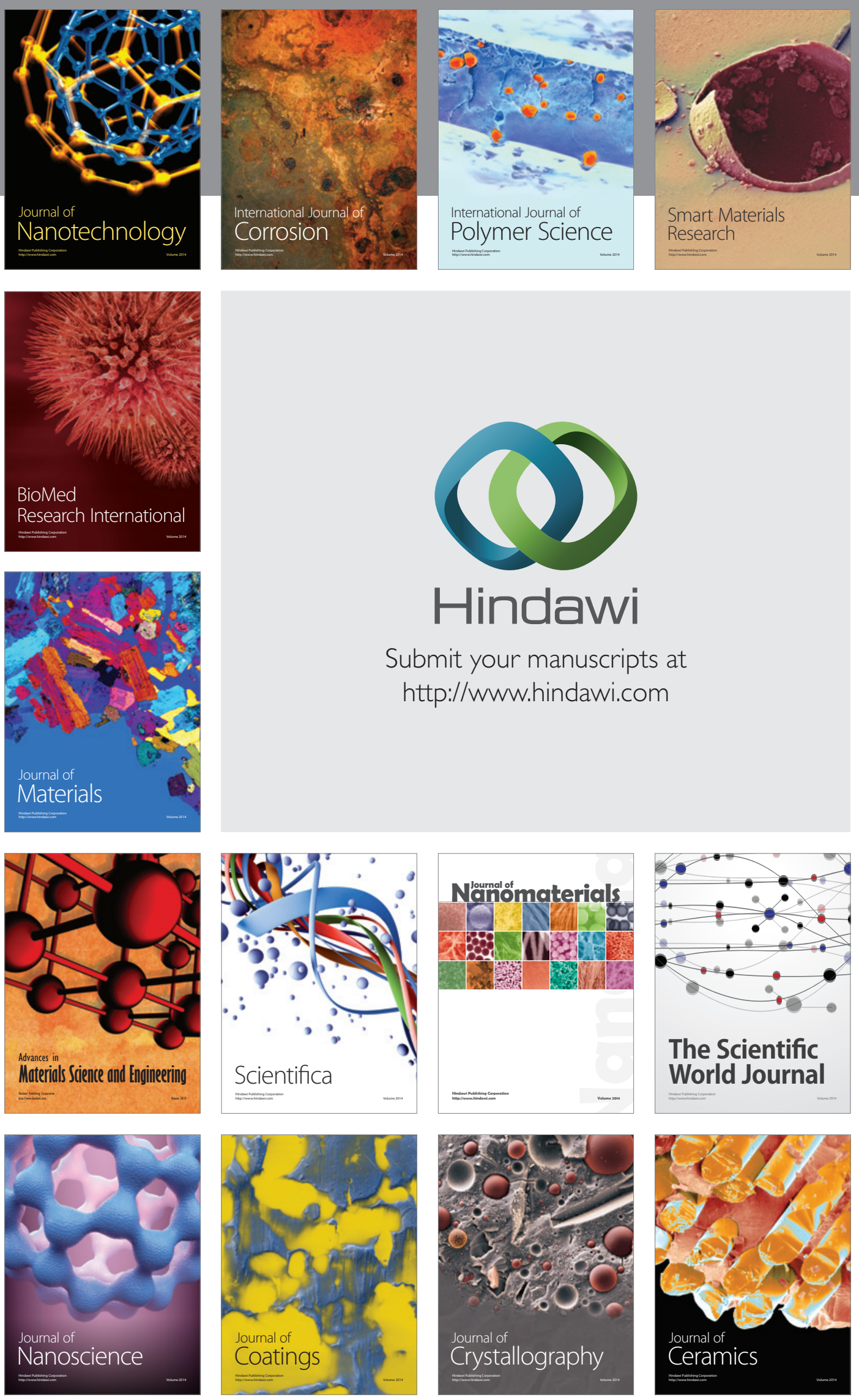

The Scientific World Journal

Submit your manuscripts at

http://www.hindawi.com

\section{World Journal}

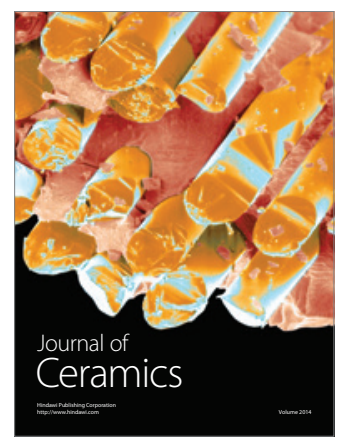

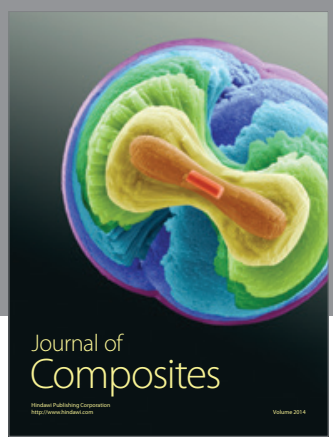
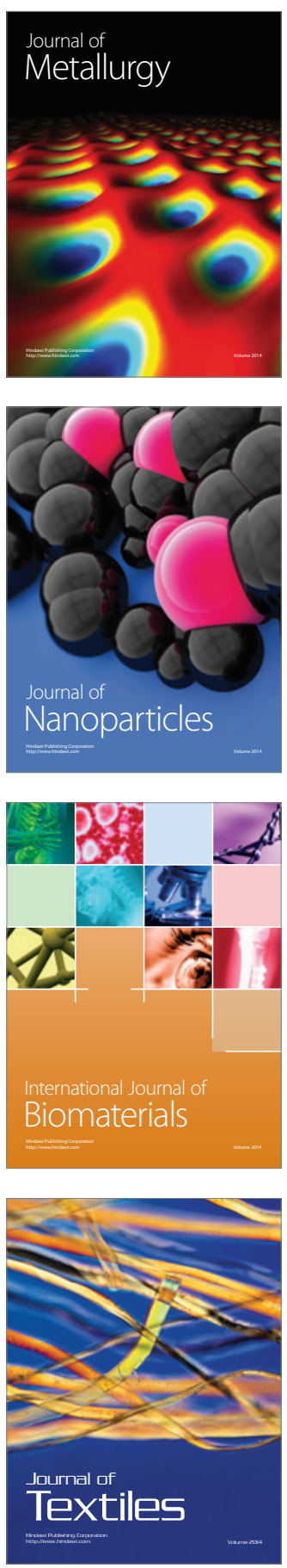